\title{
Expanded level of sympathetic chain removal does not increase the incidence or severity of compensatory hyperhidrosis after endoscopic thoracic sympathectomy
}

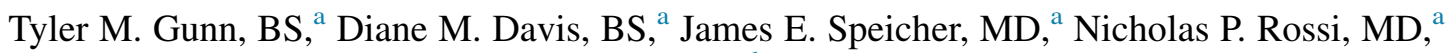
Kalpaj R. Parekh, MD, ${ }^{\mathrm{a}}$ William R. Lynch, $\mathrm{MD},{ }^{\mathrm{b}}$ and Mark D. Iannettoni, MD, $\mathrm{MBA}^{\mathrm{c}}$

\begin{abstract}
Objective: Compensatory hyperhidrosis is a common devastating adverse effect after endoscopic thoracic sympathectomy for patients undergoing surgical treatment of primary hyperhidrosis. We sought to determine whether a correlation existed in our patient population between the level and extent of sympathetic chain resection and the subsequent development of compensatory hyperhidrosis.
\end{abstract} Methods: All patients undergoing endoscopic thoracic sympathectomy in the T2-T3, T2-T4, T2-T5, or T2-T6
levels for palmar or axillary hyperhidrosis at the University of Iowa Hospital and Clinics ( $\mathrm{n}=97$ ) from January
2004 to January 2013 were retrospectively reviewed.

Results: Differences in the preoperative patient characteristics were not statistically significant among the patients receiving T2-T3, T2-T4, T2-T5, or T2-T6 level resections. Of the 97 included patients, 28 (29\%) experienced transient compensatory hyperhidrosis and $4(4 \%)$ complained of severe compensatory hyperhidrosis and required additional treatment. No operative mortalities occurred, and the morbidity was similar among the groups.

Conclusions: Most patients had successful outcomes after undergoing extensive resection without changes in the incidence of compensatory hyperhidrosis. Therefore, we recommend performing complete and adequate resection for relief of symptoms in patients with primary hyperhidrosis. (J Thorac Cardiovasc Surg 2014;148:2673-6)

Primary focal hyperhidrosis affects $1 \%$ to $3 \%$ of the population and is characterized by increased perspiration out of proportion to what is physiologically needed for thermoregulation. ${ }^{1}$ Primary focal hyperhidrosis is a chronic idiopathic condition defined as focal, visible, and excessive sweating of $\geq 6$ months' duration accompanied by 2 of the following characteristics: bilateral and symmetric symptoms, onset before age 25 years, impairment of daily activities, $\geq 1$ episode per week, focal sweating that ceases during sleep, and/or a family history of hyperhidrosis. ${ }^{2}$ It most commonly affects the palms, axillae, and plantar surfaces. ${ }^{2}$ The pathophysiology behind primary hyperhidrosis is not well understood. It has been postulated that an abnormal or exaggerated response to emotional stressors by the

\footnotetext{
From the Department of Cardiothoracic Surgery, ${ }^{a}$ University of Iowa Carver College of Medicine, Iowa City, Iowa; Section of Thoracic Surgery, ${ }^{b}$ Department of Surgery, University of Michigan, Ann Arbor, Mich; and Department of Cardiovascular Sciences, ${ }^{\text {c }}$ East Carolina Heart Institute, East Carolina University, Brody School of Medicine, Greenville, NC.

Disclosures: Authors have nothing to disclose with regard to commercial support. Received for publication Dec 20, 2013; revisions received March 3, 2014; accepted for publication June 12, 2014; available ahead of print Aug 15, 2014.

Address for reprints: Mark D. Iannettoni, MD, MBA, Department of Cardiovascular Sciences, East Carolina Heart Institute, East Carolina University, Brody School of Medicine, 115 Heart Center Dr, Greenville, NC 27834 (E-mail: Iannettonim@ecu.edu). $0022-5223 / \$ 36.00$

Copyright (C) 2014 by The American Association for Thoracic Surgery http://dx.doi.org/10.1016/j.jtcvs.2014.06.080
}

hypothalamus or cerebral cortex causes increased autonomic signaling to the eccrine sweat glands. ${ }^{3}$ Although benign in nature, hyperhidrosis can cause soaking of papers, clothes, and shoes, can lead to avoidance of social situations and limitations in professional and physical activities, and can result in emotional and psychological distress.

When topical and medical treatments have failed to resolve excessive sweating, endoscopic thoracic sympathectomy (ETS) is an effective surgical treatment of severe primary palmar hyperhidrosis, with high patient satisfaction rates. The most common devastating adverse effect after ETS is compensatory hyperhidrosis $(\mathrm{CH})$, defined as subjectively increased sweating at parts of the body not anatomically affected by the sympathectomy chain. $\mathrm{CH}$ is thought to be a thermoregulatory response by which the body compensates for the lack of perspiration at surgically denervated regions by increasing perspiration in unaffected areas, including the trunk, lumbar, groin, thigh, and popliteal regions. ${ }^{4}$ Widespread hyperhidrosis before surgery, older age, greater body mass index, high ambient temperature, and a family history of primary hyperhidrosis are risk factors for development of $\mathrm{CH}^{5,6}$ The rates of $\mathrm{CH}$ cited in published studies has ranged from $3 \%$ to $98 \%$, depending on how $\mathrm{CH}$ has been assessed. ${ }^{5}$ In attempts to decrease the incidence of $\mathrm{CH}$, many have suggested that limiting the magnitude of resection of the sympathetic chain will decrease the incidence of postoperative $\mathrm{CH}$. However, 


\section{Abbreviations and Acronyms \\ $\mathrm{CH}=$ compensatory hyperhidrosis \\ ETS $=$ endoscopic thoracic sympathectomy}

controversy remains regarding the appropriate level and number of ganglia to be removed for the best outcome. Our aim was to review the series of ETS cases performed at our institution to determine whether the level of resection influences the long-term results or overall outcomes of the procedure.

\section{METHODS}

From January 2004 to January 2013, all patients undergoing ETS in the T2-T3, T2-T4, T2-T5, and T2-T6 levels for palmar or axillary hyperhidrosis at the University of Iowa Hospital and Clinics $(\mathrm{n}=97)$ were included in a retrospective chart review. A total of 7 patients underwent ETS at levels different from the 4 groups, none of whom reported $\mathrm{CH}$, were omitted from the present study owing to an inadequate sample size. The data obtained included patient demographics, family history, site of primary hyperhidrosis, previous therapies, surgical details, outcome, and complications. The patient demographics are listed in Table 1. Of the 90 patients, 39 (40\%) experienced widespread hyperhidrosis with excessive perspiration at the palmar, axillary, and plantar locations. Of the 97 total operations, 96 were bilateral ETS. One patient underwent a planned staged procedure; he had previously undergone right-sided ETS, and only left-sided ETS was performed at our institution. The level of sympathetic ganglia removal is listed in Table 2. Seven different surgeons performed the operations.

\section{Operative Technique}

ETS is performed as a bilateral procedure with the patient in the lateral decubitus position using single lung ventilation with a double lumen tube. The arm is mildly hyperextended for exposure of the axillae. Three $5-\mathrm{mm}$ thoracoscopic ports are used, with 2 placed in the axillae, 1 at the level of the hairline for the highest port and the other in the fifth intercostal space in the mid-axillary line. After the lung has been deflated, the lowest port is inserted with a stab incision without the use of electrocautery or spreading to prevent skin damage. The other 2 ports are placed in the axillae, with the highest port placed first to not obstruct the view for placement of the third port, 1 rib space below the highest port. The lung is manipulated and compressed away from the spine to provide visualization of the sympathetic chain. The first rib is identified, and the second through sixth ribs are marked with electrocautery 3 to $5 \mathrm{~cm}$ away from the chain laterally to ensure that the level of resection stays below the first rib.

Next, the lowest level is identified, and the pleura immediately lateral to the chain is opened from the lowest to the highest point. The lowest point of the chain is then isolated and grasped with the 5-mm grasper proximally and divided with electrocautery distally. With traction in a cranial and anterior direction, the chain is gently removed using electrocautery to separate the chain from the surrounding tissues and the intercostal vessels and attachments.

Each ganglion is dissected using cautery until the second ganglion is reached. The chain is then divided just below the second rib after removing the ganglion sharply with scissors.

The area is monitored for hemostasis, and the pleura that laterally overlies the rib is superficially incised for a distance of 4 to $5 \mathrm{~cm}$ from the chain laterally with electrocautery to divide any accessory nerves that might remain. A 14F rubber catheter with multiple holes is inserted into the chest, and the lung is reinflated, with suction applied to the catheter to allow full re-expansion of the lung. The tube is then withdrawn on suction and with positive pressure of $30 \mathrm{~cm} \mathrm{H}_{2} \mathrm{O}$ applied to the lungs. The wounds are then closed with tissue adhesives. A similar procedure is performed on the other side. No chest tube or drain is used. The patient is discharged after a chest radiograph to document pulmonary re-expansion.

\section{Statistical Analysis}

Univariate comparisons of the preoperative variables were performed among the ETS T2-T3, T2-T4, T2-T5, and T2-T6 levels. Categorical variables were tested using the chi-square test, and continuous variables were assessed using the analysis of variance test for associations. Univariate comparison of the postoperative variables, including the incidence of $\mathrm{CH}$, was not performed owing to the low sample size in the T2-T3 and T2-T4 resection groups. All analyses were performed using SPSS Statistics, version 21 (IBM Corp, Armonk, NY).

\section{RESULTS}

A total of 97 patients receiving ETS for palmar or axillary hyperhidrosis were included in the present study. The sample consisted of 60 women and 37 men, with an average age of 24 years (range, 13-66). The patients had hyperhidrosis at $\geq 1$ sites, including 85 with palmar $(88 \%)$, 65 with axillary $(67 \%)$, and 61 with plantar (63\%) sites. Twelve patients $(12 \%)$ reported a family history of hyperhidrosis. All patients had been treated before surgery with medical management. This included oral glycopyrrolate (Robinul), topical aluminum chloride hexahydrate 20\% (Drysol), iontophoresis with Drionic (General Medical Co, Pasadena, Calif), injections of Botulinum toxin A, and oral clonidine. The medical interventions had failed in all patients included in the present study. The differences in the measured preoperative variables among the 4 groups were not statistically significant.

Sympathetic levels T2-T6 were removed in 41 patients $(42 \%)$, T2-T5 in 36 patients (37\%), T2-T4 in 11 patients $(11 \%)$, and T2-T3 in 9 patients $(9 \%)$. No mortalities occurred. The length of stay was $<23$ hours for all patients. All patients experienced resolution of palmar hyperhidrosis. Additionally, of the 61 patients with plantar hyperhidrosis, 7 experienced complete resolution of symptoms, and 4 reported decreased symptoms. The morbidities included 4 cases of pneumothorax, 1 episode of subcutaneous emphysema, in addition to $\mathrm{CH}$. The pneumothorax and subcutaneous emphysema resolved spontaneously without chest tube placement. No cases of Horner syndrome, excessive bleeding, or infection were reported. Most patients were satisfied with the results of the procedure.

$\mathrm{CH}$ was characterized as transient or severe. Transient $\mathrm{CH}$ was defined as a minor increase in sweating present for $<90$ days at any level, and severe $\mathrm{CH}$ was defined as persistent sweating for $\geq 90$ days requiring additional treatment. Of the 97 patients, 28 (29\%) experienced transient $\mathrm{CH}$. The breakdown of patients who experienced $\mathrm{CH}$ and the location of transient $\mathrm{CH}$ stratified by the level of sympathectomy is listed in Table 2. 
TABLE 1. Preoperative patient characteristics stratified by sympathectomy level

\begin{tabular}{|c|c|c|c|c|c|}
\hline Variable & $\begin{array}{r}\text { T2-T3 } \\
(\mathbf{n}=9)\end{array}$ & $\begin{array}{c}\text { T2-T4 } \\
(\mathrm{n}=11) \\
\end{array}$ & $\begin{array}{c}\text { T2-T5 } \\
(n=36) \\
\end{array}$ & $\begin{array}{c}\text { T2-T6 } \\
(n=41) \\
\end{array}$ & $\begin{array}{c}P \\
\text { value } \\
\end{array}$ \\
\hline Female sex & $6(67)$ & $5(45)$ & $21(58)$ & $28(68)$ & .522 \\
\hline Age (y) & $17(13-32)$ & $20(14-36)$ & $21(13-59)$ & $21(13-66)$ & .288 \\
\hline BMI $\left(\mathrm{kg} / \mathrm{m}^{2}\right)$ & $22(20-28)$ & $25(19-33)$ & $25(18-47)$ & $24(20-36)$ & .398 \\
\hline Family history & $2(22)$ & $1(9)$ & $4(11)$ & $5(12)$ & .888 \\
\hline \multicolumn{6}{|l|}{ Primary symptoms } \\
\hline Axillary & $6(67)$ & $6(55)$ & $24(67)$ & $29(71)$ & .787 \\
\hline Palmar & $8(89)$ & $10(91)$ & $30(83)$ & $37(90)$ & .803 \\
\hline Plantar & $7(78)$ & $6(55)$ & $23(64)$ & $25(61)$ & .755 \\
\hline Widespread & $5(56)$ & $3(27)$ & $13(36)$ & $18(44)$ & .546 \\
\hline \multicolumn{6}{|l|}{ Previous treatment } \\
\hline Botulinum toxin & $1(11)$ & $8(73)$ & $11(31)$ & $20(49)$ & .235 \\
\hline Iontophoresis & $4(44)$ & $2(18)$ & $10(28)$ & $11(27)$ & .622 \\
\hline
\end{tabular}

Data presented as median (range) for continuous variables or $\mathrm{n}(\%)$ for categorical variables. $B M I$, Body mass index.

Four patients $(4 \%)$ complained of severe $\mathrm{CH}$ and required additional treatment. One patient with T2-T6 ETS had $\mathrm{CH}$ affecting the abdomen, lower back, and thighs, with extremely dry hands, axillae, and upper torso. Documentation of the patient's subsequent medical treatment was unavailable. Three patients with T2-T5 ETS experienced severe $\mathrm{CH}$. The first had severe $\mathrm{CH}$ from the nipple line to the feet that was managed postoperatively with oral glycopyrrolate, topical aluminum chloride hexahydrate $20 \%$, and multiple Botulinum toxin A injections. The second and third patients with severe $\mathrm{CH}$ reported sweating on the trunk, requiring additional treatment with oral glycopyrrolate and topical aluminum chloride hexahydrate $20 \%$.

\section{DISCUSSION}

For patients with medically refractory palmar hyperhidrosis, ETS will successfully resolve the symptoms in almost every patient. ${ }^{7}$ The most devastating common complication after ETS is $\mathrm{CH}$, with an incidence reported at $3 \%$ to $98 \%$. $^{5}$ Because the goal of ETS is to improve the patient's quality of life, complications should be minimized or eliminated. Therefore, many have offered recommendations to eliminate $\mathrm{CH}$. However, controversy remains regarding the specific surgical technique, level of resection, and number of levels resected to minimize $\mathrm{CH}$ while maximizing the beneficial outcomes.

In the present study, 97 patients underwent ETS after their hyperhidrosis had been deemed refractory to medical management. Most patients at our institution received either a T2-T6 or T2-T5 resection. This was more extensive than that performed at many other institutions, which generally resect or ablate 1 to 2 levels of the sympathetic chain. Despite our larger resection area, the rate of transient $\mathrm{CH}$ at our institution was $29 \%$, similar to that reported in
TABLE 2. Operative outcomes stratified by sympathectomy level

\begin{tabular}{lcccc}
\hline \multicolumn{1}{c}{ Variable } & $\begin{array}{c}\text { T2-T3 } \\
(\mathbf{n}=\mathbf{9})\end{array}$ & $\begin{array}{c}\text { T2-T4 } \\
(\mathbf{n}=\mathbf{1 1})\end{array}$ & $\begin{array}{c}\text { T2-T5 } \\
(\mathbf{n}=\mathbf{3 6})\end{array}$ & $\begin{array}{c}\text { T2-T6 } \\
(\mathbf{n}=\mathbf{4 1})\end{array}$ \\
\hline Severe CH & $0(0)$ & $0(0)$ & $3(8)$ & $1(2)$ \\
Transient CH & $3(33)$ & $4(36)$ & $12(33)$ & $9(22)$ \\
Location of transient CH & & & & \\
$\quad$ Chest & $0(0)$ & $1(9)$ & $4(11)$ & $1(2)$ \\
Abdomen & $3(33)$ & $3(27)$ & $7(19)$ & $7(17)$ \\
Lumbar region & $3(33)$ & $2(18)$ & $10(27)$ & $4(10)$ \\
Buttocks & $0(0)$ & $0(0)$ & $2(6)$ & $0(0)$ \\
Thighs & $0(0)$ & $1(9)$ & $5(14)$ & $2(5)$ \\
Popliteal & $1(11)$ & $0(0)$ & $1(3)$ & $1(2)$ \\
\hline
\end{tabular}

Data presented as $\mathrm{n}(\%) . \mathrm{CH}$, Compensatory hyperhidrosis.

published studies of patient populations undergoing resection of only 1 or 2 ganglia. ${ }^{8-10}$ Similarly, the rate of severe $\mathrm{CH}$ at our institution was $4.1 \%$, in keeping with the lowest rates reported in published studies.

We defined severe $\mathrm{CH}$ as persistent excessive perspiration lasting $>90$ days after surgery that required additional treatment. In our experience, many patients will complain of minor increased sweating in the initial weeks and months after surgery. Patients with complaints of $\mathrm{CH}$ are given an appointment 1 month after ETS and then asked to followup at 3 and 6 months after ETS. Most of the patients with $\mathrm{CH}$ will cancel their follow-up appointments because their $\mathrm{CH}$ resolves spontaneously.

It has been postulated that $\mathrm{CH}$ is a thermoregulatory response of the remaining sweat glands after denervation of many of the upper extremity sweat glands by ETS. ${ }^{4}$ Many suggestions have been made to limit the number of levels affected by ETS as much as possible. It was initially thought that fibers from $\mathrm{T} 2$ alone innervate the eccrine sweat glands in the upper extremity; thus, it was recommended that T2 alone be divided. ${ }^{8}$ However, it was then discovered that many patients have postganglionic nerves, termed "accessory nerves of Kuntz," leaving the sympathetic chain at the T3 or T4 level and entering the brachial plexus to innervate the upper extremity. This led to recommendations to denervate, not only $\mathrm{T} 2$, but also $\mathrm{T} 2-\mathrm{T} 3$ or, even, T2-T4. ${ }^{8}$

In a 2009 review of 282 patients who underwent ETS, Miller and colleagues ${ }^{6}$ advocated division of the sympathetic chain at the T2 level only. They reported a significant decrease in $\mathrm{CH}$ in the $\mathrm{T} 2$ division group compared with the $\mathrm{T} 2-\mathrm{T} 4$ group with multiple divisions of the chain. Of the $\mathrm{T} 2$ and T2-T4 groups $13 \%$ and $34 \%$ experienced $\mathrm{CH}$, respectively. ${ }^{6}$ In contrast, from the results of a 2009 prospective randomized control study of 60 patients undergoing ETS by division and thermoablation of either the T2 or T3 ganglion, Yazbek and colleagues ${ }^{8}$ recommended division of the $\mathrm{T} 3$ ganglion only to reduce the incidence of $\mathrm{CH}$. By 20 months of follow-up, all the patients in their study reported some degree of $\mathrm{CH}$. The patients in the $\mathrm{T} 2$ group had significantly more severe 
(defined as visible, embarrassing, and requiring a change of clothing) and nonsevere $\mathrm{CH}$ than those in the $\mathrm{T} 3$ group. However, no statistically significant difference was found in patient-reported quality of life at 1,6 , or 20 months. ${ }^{8}$

Others have suggested interruption of the sympathetic chain in multiple locations. In 2011, the Society of Thoracic Surgeons conducted a review of $>400$ studies. They concluded that patients with palmar hyperhidrosis should undergo T3 and/or T4 sympathectomy; patients with T3 sympathectomy had increased $\mathrm{CH}$, and patients with $\mathrm{T} 4$ sympathectomy had incomplete resolution of palmar sweating. For patients with widespread hyperhidrosis at the palmar, axillary, and plantar sites, the Society of Thoracic Surgeons has recommended T4 and T5 sympathectomy. ${ }^{5}$

Several studies have shown that division of $\mathrm{T} 2, \mathrm{~T} 3$, or T4 or some combination reduces the incidence of $\mathrm{CH}$. However, many studies have suggested that $\mathrm{CH}$ occurs in many patients undergoing sympathectomy for hyperhidrosis, regardless of the division level. Katara and colleagues ${ }^{10}$ reported on 25 patients who had undergone T2 division on 1 side and T2-T3 division on the other side. These patients all experienced resolution of their symptoms bilaterally, and $80 \%$ developed $\mathrm{CH}$ bilaterally, suggesting that the level of chain interruption did not affect the development of $\mathrm{CH} .{ }^{10}$ Similarly, Leseche and colleagues ${ }^{11}$ reviewed 134 patients with multiple different levels of resection from T1 to T5 and noted no significant correlation between the extent of resection and the incidence or severity of $\mathrm{CH}$. Chwajol and colleagues ${ }^{12}$ also reviewed patients undergoing various levels of resection from $\mathrm{T} 2$ to $\mathrm{T} 4$ and concluded that no difference was present in the incidence of $\mathrm{CH}$ by the level of resection. The only difference they noted was a significant decrease in severe, debilitating $\mathrm{CH}$ in the $\mathrm{T} 3$ resection group. ${ }^{12}$ In a 2008 review of 87 studies, Kopelman and Hashmonai ${ }^{13}$ studied the effect of multiple combinations of $\mathrm{T} 1, \mathrm{~T} 2, \mathrm{~T} 3, \mathrm{~T} 4$, and $\mathrm{T} 5$ sympathectomy by various techniques. They concluded that limiting the level of sympathetic ablation did not decrease the incidence of $\mathrm{CH}^{13}$ Thus, in several relatively large studies considering multiple combinations of different chain resections, no difference was found in the incidence of $\mathrm{CH}$.

The primary shortcomings of our study were the retrospective nature and the small sample size for the T2-3 and T2-T4 groups. The patients in the present study were not randomized to a resection group and paired for analysis, limiting direct statistical comparisons. Additionally, the disparity in the number of patients among the resection groups negatively affected meaningful statistical analysis. As mentioned, the extent of ganglion resection in our pa- tients was larger than in many other studies; however, the incidence of transient and severe $\mathrm{CH}$ was similar to that consistently reported in published studies among those advocating for resection of only 1 or 2 ganglia.

\section{CONCLUSIONS}

The results of the present retrospective study have indicated that in our patient population little difference was present in the outcomes or incidence of $\mathrm{CH}$, regardless of the level or extent of resection. Most of our patients underwent extensive resection, with a successful outcome and without a change in the incidence of $\mathrm{CH}$. Therefore, we do not recommend limiting the resection level in an attempt to limit the incidence or severity of $\mathrm{CH}$. Because the extent of resection had no effect on the occurrence of $\mathrm{CH}$ and other complications, we recommend performing a complete and adequate resection for relief of symptoms in patients with primary hyperhidrosis.

\section{References}

1. Strutton DR, Kowalski JW, Glaser DA, Stang PE. US prevalence of hyperhidrosis and impact on individuals with axillary hyperhidrosis: results from a national survey. J Am Acad Dermatol. 2004;51:241-8.

2. Hornberger J, Grimes K, Naumann M, Glaser DA, Lowe NJ, Naver H, et al. Recognition, diagnosis, and treatment of primary focal hyperhidrosis. J Am Acad Dermatol. 2004;51:274-86.

3. Sato K, Kang WH, Saga K, Sato KT. Biology of sweat glands and their disorders. II. Disorders of sweat gland function. J Am Acad Dermatol. 1989;20:713-26.

4. Cramer MN, Jay O. Compensatory hyperhidrosis following thoracic sympathectomy: a biophysical rationale. Am J Physiol Regul Integr Comp Physiol. 2012; 302:R352-6.

5. Cerfolio RJ, De Campos JR, Bryant AS, Connery CP, Miller DL, DeCamp MM, et al. The Society of Thoracic Surgeons expert consensus for the surgical treatment of hyperhidrosis. Ann Thorac Surg. 2011;91:1642-8.

6. Miller DL, Bryant AS, Force SD, Miller JI Jr. Effect of sympathectomy level on the incidence of compensatory hyperhidrosis after sympathectomy for palmar hyperhidrosis. J Thorac Cardiovasc Surg. 2009;138:581-5.

7. Panhofer P, Zacherl J, Jakesz R, Bischof G, Neumayer C. Improved quality of life after sympathetic block for upper limb hyperhidrosis. Br J Surg. 2006;93:582-6.

8. Yazbek G, Wolosker N, Kauffman P, Campos JR, Puech-Leao P, Jatene FB. Twenty months of evolution following sympathectomy on patients with palmar hyperhidrosis: sympathectomy at the T3 level is better than at the T2 level. Clinics (Sao Paulo). 2009;64:743-9.

9. Li X, Tu YR, Lin M, Lai FC, Chen JF, Dai ZJ. Endoscopic thoracic sympathectomy for palmar hyperhidrosis: a randomized control trial comparing T3 and T2 4 ablation. Ann Thorac Surg. 2008;85:1747-51.

10. Katara AN, Domino JP, Cheah WK, So JB, Ning C, Lomanto D. Comparing T2 and T2-T3 ablation in thoracoscopic sympathectomy for palmar hyperhidrosis: a randomized control trial. Surg Endosc. 2007;21:1768-71.

11. Leseche G, Castier Y, Thabut G, Petit MD, Combes M, Cerceau O, et al. Endoscopic transthoracic sympathectomy for upper limb hyperhidrosis: limited sympathectomy does not reduce postoperative compensatory sweating. J Vasc Surg. 2003;37:124-8.

12. Chwajol M, Barrenechea IJ, Chakraborty S, Lesser JB, Connery CP, Perin NI. Impact of compensatory hyperhidrosis on patient satisfaction after endoscopic thoracic sympathectomy. Neurosurgery. 2009;64:511-8; discussion 518.

13. Kopelman D, Hashmonai M. The correlation between the method of sympathetic ablation for palmar hyperhidrosis and the occurrence of compensatory hyperhidrosis: a review. World J Surg. 2008;32:2343-56. 